\title{
Long-acting nifedipine in the management of the hypertensive patient
}

\author{
Morgan E Snider' \\ Donald S Nuzum² \\ Angie Veverka ${ }^{2}$ \\ 'Virginia Commonwealth University \\ Health Systems Richmond, VA \\ USA; ${ }^{2}$ Wingate University School \\ of Pharmacy Wingate, NC USA
}

\begin{abstract}
Hypertension is a global condition affecting billions worldwide. It is a significant contributor to cardiovascular events, cardiac death and kidney disease. A number of medication classes exist to aid healthcare providers and their patients in controlling hypertension. Nifedipine, a dihydropyridine calcium channel blocker, was once one of the most widely used medications for hypertension, but safety and tolerability concerns along with the introduction of new classes of antihypertensive medications and an increasing pool of data showing mortality benefit of other classes caused nifedipine to fall out of favor. More recently, long-acting formulations were developed and made available to clinicians. These newer formulations were designed to address many of the concerns raised by earlier formulations of nifedipine. Numerous clinical trials have been conducted comparing long-acting nifedipine to many of the more commonly prescribed antihypertensive medications. This review will address the pharmacology, pharmacokinetics and the available clinical trial data on long-acting nifedipine and summarize its role in the management of hypertension.
\end{abstract}

Keywords: nifedipine, calcium channel blockers, hypertension

\section{Introduction}

Hypertension is a progressive disease that affects more than 1 billion people around the world (Chobanian et al 2003). The risk of developing hypertension increases with age and according to the Framingham Heart Study, even those who have a normal blood pressure (BP) at the age of 55 still carry a lifetime risk for developing hypertension of $90 \%$ (Vasan et al 2002). Over time, untreated or poorly controlled hypertension can lead to acute illness such as myocardial infarction and stroke (Lewington et al 2002). Long-standing hypertension is also a risk factor for chronic comorbidities ranging from coronary artery disease to kidney disease to left ventricular hypertrophy and heart failure (Lewington et al 2002; Rosendorff et al 2007).

American and European guidelines recommend first-line agents for the treatment of hypertension based on the patient's varying "compelling indications" or comorbid disease states (Chobanian et al 2003; Mancia et al 2007). Because several classes of medications, such as angiotensin converting enzyme inhibitors (ACEIs) and $\beta$-blockers, are appropriate for numerous compelling indications their use predominates other classes that are less universal, such as calcium channel blockers (CCBs). Dihydropyridine CCBs have been determined to be appropriate for first-line therapy in patients with hypertension, particularly in those with left ventricular hypertrophy, asymptomatic atherosclerosis, angina pectoris, permanent atrial fibrillation, peripheral artery disease, isolated systolic hypertension, metabolic syndrome, and pregnancy (Lewington et al 2002; Blood Pressure Lowering Treatment Trialists' Collaboration 2003; Staessen et al 2003; Mancia et al 2007). Non-dihydropyridine CCBs are also appropriate for use in patients with angina pectoris and carotid atherosclerosis (Mancia et al 2007). One trial found no significant difference between ACEIs and CCBs in terms of total 
number of coronary events, cardiovascular mortality, total mortality, or coronary heart disease (Blood Pressure Lowering Treatment Trialists' Collaboration 2003). Extended-release calcium channel blockers are recommended as an appropriate first-line agent for the treatment of ischemic heart disease, particularly in patients with stable angina pectoris (Chobanian et al 2003). Other potential indications include those patients at an increased risk for coronary disease and diabetes, where CCBs have been shown to reduce the incidence of cardiovascular disease and stroke, Raynaud's syndrome, and specific arrhythmias (Chobanian et al 2003). CCBs have not been shown to prevent the incidence of heart failure (Blood Pressure Lowering Treatment Trialists' Collaboration 2003). African American patients tend to have an increased response to CCBs and diuretics as compared to other antihypertensives such as ACEIs, angiotensin receptor blockers (ARBs), and $\beta$-blockers (The ALLHAT Officers and Coordinators for the ALLHAT Collaborative Research Group 2002). It is important to note that not all CCBs are alike and in fact this class is more heterogenous than most other classes of antihypertensives therefore each agent needs to be considered individually.

Nifedipine is a dihydropyridine calcium channel blocker, the short acting formulation of which has been associated with reflex sympathetic nervous system (SNS) activation leading to flushing, tachycardia, worsening myocardial ischemia, and cerebrovascular ischemia; therefore only longer acting formulations should be used (Gibbons et al 2003). Numerous extended-release formulations are available worldwide and have been shown to be equally efficacious as compared to other antihypertensives such as ARBs, $\beta$-blockers, and diuretics in the management of hypertension (Frishman et al 1987; Weir et al 1996; Brown et al 2000). This review focuses on the extended-release formulations of nifedipine and their role in the treatment of patients with hypertension.

\section{Pharmacology}

Nifedipine exerts its effect in hypertension, as well as angina, by acting as an arterial vasodilator. Calcium ions regulate smooth muscle contractions contributing to inotropic and chronotropic activity in the heart (Rosendorff et al 2007). The L-type channels in vascular smooth muscle permit the entrance of calcium ions which potentiates a contraction (Abernathy et al 1999). Dihidropyridine CCBs such as nifedipine bind to the L-type channel in arterial tissue, particularly coronary arteries, preventing the influx of calcium ions which allows for vasodilation, thus increasing myocardial oxygen supply (Abrams et al 2001; Pfizer 2003). Myocardial oxygen demand is reduced with the decrease in peripheral vascular resistance (Abrams et al 2001). CCBs are also responsible for a decrease in afterload, illustrated by a decrease in systolic BP (SBP) (Abrams et al 2001). The decrease in BP depends on the baseline value such that patients with a higher BP will experience a more significant reduction (Frishman et al 1989). Several studies have also shown a decrease in the development of new atherosclerotic lesions with the use of dihydropyridine CCBs which is attributed to their vascular protective characteristics (Wenzel et al 1997).

\section{Pharmacokinetics}

Nifedipine displays zero-order kinetics across the dosing range from $30 \mathrm{mg}$ to $180 \mathrm{mg}$ with an estimated elimination half-life of 1.7 hours. This is significant considering the effect on heart rate (HR) and BP corresponds to plasma drug concentration (Swanson et al 1987; Pfizer 2003). Renal impairment does not affect the half-life of nifedipine unless it is severe $(\mathrm{CrCl}<25 \mathrm{~mL} /$ minute $)$, in which case the half-life is extended to approximately 3.8 hours (Chung et al 1987). Sixty to eighty percent of the dose is excreted as an inactive metabolite in the urine (Pfizer 2003). Nifedipine is hepatically metabolized and is $92 \%$ to $98 \%$ protein bound. Due to significant first-pass metabolism nifedipine's bioavailability is between 45\%-68\% (Chung et al 1987; Pfizer 2003). Chronic liver disease may prolong the disposition half-life and increase the bioavailability (Pfizer 2003).

Extended-release formulations of nifedipine are desired in order to prolong the therapeutic effect and prevent reflex SNS activation. The nifedipine gastrointestinal therapeutic system (GITS) is one of the more prevalent extended-release formulations. There are two layers in the system; the top contains drug and osmotic agent and the bottom containing an osmotic driving agent only (Chung et al 1987; Swanson et al 1987). Both layers take water in across the membrane via osmosis creating a suspension within the top layer (Swanson et al 1987). This suspension is driven out through a pre-drilled hole as the osmotic layer expands (Swanson et al 1987). The rate at which drug disperses corresponds to the rate at which water enters the system; generally speaking, drug is released consistently over 16 to 18 hours with a bioavailability between $75 \%$ and $85 \%$ at steady state (Chung et al 1987 ; Swanson et al 1987). The diameter of the pre-drilled opening limits the rate at which drug exits the system, thus preventing a dose-dumping effect (Swanson et al 1987; Chung et al 1987). This process does not depend upon $\mathrm{pH}$ or intestinal 
motility, therefore drug distribution out of the system does not vary with gastrointestinal contents or function (Swanson et al 1987). The exception occurs when retention time in the gut is extensively minimized to less than 24 hours, such as with short bowel syndrome, in which case the full dose is not absorbed (Chung et al 1987).

Studies have been conducted that compare various nifedipine formulations in an attempt to establish if there is a clinically significant benefit of one extended-release formulation over another. It has been established that the reflex activation of the SNS correlates with the rate of increase in plasma drug levels. Thus it is hypothesized that a more gradual rise in drug concentrations should decrease SNS activation, in turn reducing adverse events associated with short acting nifedipine (Fogari et al 2003). One such trial of 25 patients compared nifedipine GITS $60 \mathrm{mg}$ once daily (Adalat $\mathrm{XL}^{\circledR}$; Bayer) to either nifedipine prolonged action $20 \mathrm{mg}$ twice daily (Adalat $\mathrm{PA}^{\circledR}$ ) or two $10 \mathrm{mg}$ nifedipine capsules every 8 hours (Adalat ${ }^{\circledR}$ ) under fasting conditions (Toal et al 2004). This trial failed to demonstrate a relationship between peak plasma drug concentrations with the three formulations and BP reduction, which opposes the findings of previous studies (Toal et al 2004). The author concluded that patients should not be switched between formulations because although there was no correlation with BP, peak plasma drug concentrations may correspond to reflex activation of the SNS, thus inducing cardiovascular events (Toal et al 2004).

Due to the high incidence of tachycardia and vasodilatory symptoms associated with short-acting nifedipine, emphasis has been placed on determining the effect of extended-release nifedipine on catecholamine levels and corresponding HR and BP values. It has been suggested that a higher incidence of cardiovascular events associated with nifedipine may be due to reflex activation of the SNS (Champlain et al 1998). As long-acting nifedipine formulations became more readily available attention was placed on the occurrence of reflex SNS activation in correlation with plasma drug concentration (Champlain et al 1998). One study compared the acute and chronic effects of nifedipine on HR, BP, norepinephrine (NE), and epinephrine levels (Champlain et al 1998). Sixtyfive patients with mild to moderate hypertension were treated with nifedipine retard $10 \mathrm{mg}$ twice daily, nifedipine GITS $30 \mathrm{mg}$ daily, or amlodipine $5 \mathrm{mg}$ daily for 2 weeks with the option to double treatment doses for the last 4 weeks if BP was not controlled (Champlain et al 1998). Acute treatment effects were assessed just before and after the first treatment dose whereas chronic effects were analyzed after 6 weeks of therapy (Champlain et al 1998). All three treatment arms significantly reduced SBP and diastolic BP (DBP) at 2 weeks through the end of the treatment period, but only amlodipine was found to increase HR significantly by the end of therapy ( $75 \pm 2$ beats per min to $81 \pm 2.3$ beats per minute, $p<0.05$ ) While all three treatments equally reduced BP, their effects on norepinephrine levels were not equivalent. Plasma norepinephrine levels increased significantly with nifedipine retard, both acutely and chronically, reaching their peak 3 hours after drug administration (Champlain et al 1998). Similarly, chronic amlodipine therapy increased plasma levels of norepinephrine by 50\% compared to baseline (Champlain et al 1998). Chronic therapy with nifedipine GITS decreased plasma NE levels significantly 6 hours after the dose and this effect was maintained throughout the dosing interval (Champlain et al 1998). The nifedipine GITS formulation was associated with a more gradual decrease in BP without an increase in NE, potentially due to the fact that plasma drug levels were lower than those obtained by nifedipine retard (Champlain et al 1998). This study demonstrated a relationship between an increase in plasma norepinephrine, the rate of increase in drug concentration, and a subsequent and sudden drop in BP (Champlain et al 1998).

Another, 48-week study compared nifedipine GITS to lercanidipine, a third generation $\mathrm{CCB}$ whose lipophilicity allows for a longer half-life (Fogari et al 2003). Patients were randomized to $10 \mathrm{mg}$ of lercanidipine or $30 \mathrm{mg}$ of nifedipine GITS for 4 weeks, after which the doses could be doubled if the patient's DBP was $>90 \mathrm{mmHg}$ (Fogari et al 2003). BP, $\mathrm{HR}$, and NE levels were evaluated throughout the study period (Fogari et al 2003). Compared to placebo, both treatments effectively decreased BP ( $p<0.001$ for both arms) with no significant change in HR (Fogari et al 2003). There was a significant increase in NE concentrations at 4 weeks in both treatment arms (Fogari et al 2003). Only nifedipine GITS significantly increased NE levels at 48 weeks compared to baseline drug concentrations reached their peak and 12 hours after the dose ( $p<0.05$ at both times) (Fogari et al 2003). Because HR was not affected despite changes in NE, this suggests the effects of nifedipine GITS on the SNS might be selective for peripheral and not cardiac nerves (Fogari et al 2003). Just as BP control is a predictor for cardiovascular events, norepinephrine levels are a similar surrogate marker; therefore, because both agents decreased BP effectively, little can be extrapolated from the variation in effect on norepinephrine.

\section{Clinical trials}

Nifedipine has been compared head-to-head with several other antihypertensives, particularly when the GITS formulation 
was released. With a diminished concern for reflex SNS activation nifedipine had the potential to play a larger role in the management of hypertension. In a 10-week, multi-center, double-blind study of 102 participants, patients received nifedipine GITS 30 or $60 \mathrm{mg}$ daily, hydrochlorothiazide (HCTZ) 25 or $50 \mathrm{mg}$ daily, or placebo (Gavras et al 1987). The majority of patients in the active treatment arms finished the study on $50 \mathrm{mg}$ of HCTZ or $60 \mathrm{mg}$ of nifedipine GITS (Gavras et al 1987). Both treatments were significantly better than placebo in decreasing SBP and DBP with $71 \%$ of the HCTZ group and $67 \%$ of the nifedipine group achieving a sitting DBP $<90 \mathrm{mmHg}$ (Gavras et al 1987). The authors concluded that nifedipine GITS monotherapy decreases BP with efficacy similar to that of HCTZ (Gavras et al 1987).

In another double-blind study, patients received nifedipine GITS or sustained-release propranolol for 8 weeks, both of which could be titrated to an optimal dose if the DBP remained $>90 \mathrm{mmHg}$ (Frishman et al 1989). The main objective of the trial was to evaluate the change in BP from baseline as well as the proportion of patients whose BP was decreased to goal (Frishman et al 1989). The majority of patients in the nifedipine and propranolol groups ended the trial on $90 \mathrm{mg}$ and $240 \mathrm{mg}$ daily, respectively (Frishman et al 1989). In this study, sitting SBP was decreased a mean of $15.9 \mathrm{mmHg}$ in the nifedipine group compared to $5.7 \mathrm{mmHg}$ in the propranolol group $(\mathrm{p}<0.001)$ (Frishman et al 1989); sitting DBP was reduced by a mean of $10 \mathrm{mmHg}$ in the nifedipine arm versus $6.1 \mathrm{mmHg}$ in the propranolol group $(\mathrm{p}<0.018)$ (Frishman et al 1989). Standing SBP was also reduced to a greater extent in the nifedipine group $(\mathrm{p}<0.005)$ (Frishman et al 1989). The proportion of patients receiving nifedipine who achieved a goal decrease in sitting and standing BP was $61 \%$ and $52 \%$, respectively, as compared to $25 \%$ and $28 \%$ in the propranolol group (Frishman et al 1989). This study showed nifedipine GITS to be more efficacious than sustained-release propranolol in reducing sitting SBP and DBP, as well as standing SBP (Frishman et al 1989).

Nifedipine has also been compared to other dihydropyridine CCBs, such as amlodipine. One particular study randomized patients to daily nifedipine GITS $30 \mathrm{mg}$ or amlodipine $5 \mathrm{mg}$ for 21 weeks to assess the effect on DBP as well as quality of life (Testa et al 1998). The main objectives of the study were to evaluate the change in SBP and DBP as well as the change in health-related quality of life. Results pertaining to quality of life will be discussed later (see Tolerability). The study showed no significant difference between treatment arms with respect to SBP or DBP (Testa et al 1998). The mean decrease in SBP was $18.8 \mathrm{mmHg}$ with nifedipine and
19.7 with amlodipine while the mean reduction in DBP was 15.5 with nifedipine and 15.7 with amlodipine ( $p>0.55$ for SBP and DBP) (Testa et al 1998).

As mentioned previously, ACEIs are recommended as first-line antihypertensives in patients with varying comorbid conditions. One study compared the efficacy of once-daily nifedipine GITS 30 to $60 \mathrm{mg}$ with that of enalapril 5 to $10 \mathrm{mg}$ daily over the course of 8 weeks by measuring BP and HR at each visit as well as utilizing ambulatory BP monitoring (ABPM) (Schulte et al 2000). At the end of the treatment period DBP and SBP were significantly decreased from baseline in both groups ( $\mathrm{p}<0.001$ for DBP in both treatments) (Schulte et al 2000). More patients in the nifedipine group were maintained on a low-dose regimen compared to the enalapril group ( $\mathrm{p}<0.05$ ) (Schulte et al 2000). Twenty-four hour SBP decreased from $141 \pm 15 \mathrm{mmHg}$ to $134 \pm 14 \mathrm{mmHg}$ in the nifedipine group compared to enalapril where SBP decreased from $140 \pm 15 \mathrm{mmHg}$ to $131 \pm 15 \mathrm{mmHg}$ (Schulte et al 2000). DBP was $86 \pm 9 \mathrm{mmHg}$ for both groups at baseline and decreased to $82 \pm 9 \mathrm{mmHg}$ in the nifedipine group and $80 \pm 8 \mathrm{mmHg}$ in the enalapril group (Schulte et al 2000). This trial demonstrated that nifedipine GITS and enalapril are similarly efficacious for the treatment of hypertension (Schulte et al 2000).

ARBs are a standard first-line therapy and alternative to ACEIs in the treatment of hypertension, particularly in those patients with compelling indications such as diabetes, chronic kidney disease, and heart failure (Chobanian et al 2003). One trial sought to compare the effects of nifedipine GITS $30 \mathrm{mg}$, $60 \mathrm{mg}$, or $90 \mathrm{mg}$ with losartan $50 \mathrm{mg}$ monotherapy or with HCTZ $12.5 \mathrm{mg}$ or $25 \mathrm{mg}$ on mean sitting DBP after 12 weeks (Weir et al 1996). Two hundred twenty-three patients were treated and decreases in BP were similar in both groups (Weir et al 1996). Those patients who had a higher baseline DBP (106-115 mmHg) showed a significantly greater benefit for sitting DBP at the end of therapy when treated with losartan compared with nifedipine GITS $(-16.2 \pm 7.1 \mathrm{mmHg}, \mathrm{p}=0.03)$ (Weir et al 1996). There was no significant difference in SBP, the percentage of patients who had a sitting DBP $<90 \mathrm{mmHg}$ at the end of treatment, HR, quality of life, or adverse events between the two groups (Weir et al 1996). The authors concluded that losartan is similarly efficacious to nifedipine GITS in the treatment of hypertension but with greater tolerability, particularly with respect to edema (Weir et al 1996). Similar results were found in an open-label study comparing nifedipine GITS to telmisartan in combination with HCTZ (Fogari et al 2005).

It has been well demonstrated that short-acting nifedipine can cause reflex sympathetic activation. What has been less 
studied is how extended-release nifedipine affects the SNS. Several trials have evaluated the effects of extended-release nifedipine on HR and BP throughout the dosing interval. A study by Wenzel and colleagues evaluated the effect of nifedipine GITS $60 \mathrm{mg}$ on muscle sympathetic nervous activity (MSA) as compared to short-acting nifedipine $5 \mathrm{mg}$ and $10 \mathrm{mg}$ or placebo in normotensive patients (Wenzel et al 1997). MSA, measured in change of bursts per minute, increased significantly following $10 \mathrm{mg}$ of short-acting nifedipine and $60 \mathrm{mg}$ nifedipine GITS ( $\mathrm{p}<0.05$ vs placebo). There was no significant change in HR in the nifedipine GITS group compared to baseline; however during the cold pressor test HR increased significantly $(\mathrm{p}<0.05)$. SBP did not change but DBP increased significantly following the administration of nifedipine GITS $(\mathrm{p}<0.05)$. Because plasma NE levels are a predictor of mortality in heart failure patients and elevated levels may be harmful in patients with hypertension, concentrations were evaluated at various time points in this trial. Concentrations increased significantly 150 minutes after drug administration $(\mathrm{p}<0.05)$ but plasma epinephrine levels did not change significantly. Plasma endothelin levels were significantly elevated in the nifedipine GITS group 6 hours after drug intake $(\mathrm{p}<0.05)$. This trial showed that nifedipine stimulates peripheral sympathetic nerve activity, despite the formulation of the drug; however there was no change in cardiac sympathetic activity. It is proposed that because extended-release nifedipine allows for a more controlled onset of vasodilation, the SNS is selectively activated in the periphery leading to an increase in MSA while the cardiac sympathetic system is not affected as demonstrated by the lack of change in HR (Wenzel et al 1997). A similar theory has been proposed in previously discussed studies (Fogari et al 2003).

Other cardiovascular pathways are implicated in the progression of hypertension and nifedipine may have benefits beyond its vasodilatory effects. There are several mechanistic factors involving granulocyte-macrophage colonystimulating factor (GM-CSF) and tumor necrosis factor- $\alpha$ $(\mathrm{TNF}-\alpha)$. GM-CSF and TNF- $\alpha$ are increased in patients with hypertension and this may contribute to an increased risk for atherosclerosis (Shima et al 2008). These details have just recently been elucidated in a trial that evaluated the effects of CCBs on superoxide $\left(\mathrm{O}_{2}^{-}\right)$liberation from neutrophils. $\mathrm{O}_{2}^{-}$release is mediated by GM-CSF and TNF- $\alpha$ and studies have shown that some CCBs may inhibit this process (Shima et al 2008). $\mathrm{O}_{2}^{-}$release mediated by TNF- $\alpha$ was suppressed significantly by nifedipine $(\mathrm{p}<0.01)$ (Shima et al 2008). The authors of this trial suggest that some CCBs may have a role in the prevention of atherosclerosis via the suppression of neutrophil activation (Shima et al 2008). One should keep in mind that this study looked at several surrogate endpoints for atherosclerosis in vitro, therefore the clinical significance is unknown. As this is a relatively new area of study more data are needed before the effects of nifedipine on atherosclerosis can be substantiated.

All of these trials share similar limitations in that their duration of treatment and follow-up is relatively short, ranging from 8 to 48 weeks (Frishman et al 1989; Schulte et al 2000; Fogari et al 2003). The mean age of patients in the previously mentioned studies ranges between the sixth and seventh decades of life; therefore most of these patients can expect to live another 20 to 30 years (Mancia et al 2007). Patients were followed for just a few months despite the fact that complications from poorly controlled hypertension take years to manifest. Ultimately it is the occurrence of an event, whether fatal or non-fatal, that is of interest, not the surrogate endpoints such as changes in BP from baseline. While it is well understood that BP is a strong predictor of cardiovascular events, the duration of follow up may not be adequate to determine a truly beneficial effect, or lack thereof.

A prospective, double-blind trial by Brown and colleagues assessed morbidity and mortality in European and Israeli patients who had hypertension in addition to a cardiovascular risk factor such as hypercholesterolemia, CHD, peripheral vascular disease, left ventricular hypertrophy, or a family history of myocardial infarction (Brown et al 2000). The primary endpoint of the International Nifedipine GITS Study: Intervention as a Goal in Hypertension Treatment (INSIGHT) trial was the composite of death from a cardiovascular or cerebrovascular cause, non-fatal stroke, myocardial infarction, and heart failure. Patients were randomized to receive nifedipine GITS $30 \mathrm{mg}$ or co-amilozide (HCTZ $25 \mathrm{mg}$ /amiloride $2.5 \mathrm{mg}$ ) daily (Brown et al 2000). The primary outcome was reported in $6.3 \%$ of patients in the nifedipine group compared to $5.8 \%$ in the co-amilozide group ( $p=0.34)$. There was no significant difference in event rates between groups. Significantly greater number of patients experienced a fatal myocardial infarction or nonfatal heart failure in the nifedipine group $(0.5 \%$ vs $0.2 \%$, $\mathrm{p}=0.017$ and $0.8 \%$ vs $0.3 \%, \mathrm{p}=0.028$, respectively). The authors concluded that both agents were equally efficacious in preventing cardiovascular and cerebrovascular complications (Brown et al 2000).

One subanalysis of the INSIGHT data was performed to evaluate ambulatory BP values before and after treatment (Mancia et al 2002). Office and 24-hour average SBP, 
DBP, and pulse pressure were significantly reduced by both treatments. The authors therefore concluded that nifedipine and diuretics offer similar protection against cardiovascular events (Mancia et al 2002). Another subanalysis evaluated outcomes in patients with isolated systolic hypertension (ISH) (Mancia et al 2004). Primary outcomes occurred in $6 \%$ of patients in the nifedipine arm compared to $6.6 \%$ in the group receiving diuretics $(p=0.67)$ (Mancia et al 2004). There were no significant differences in the composite endpoint between those patients with ISH compared to non-ISH patients (Mancia et al 2004). According to logistic regression analyses ISH was not found to be a predictor of the composite primary outcomes after adjusting for baseline characteristics (Mancia et al 2004). The study's authors determined that nifedipine GITS and diuretics are equally efficacious in the treatment of patients with ISH (Mancia et al 2004).

\section{Chronotherapy}

It has been well documented that HR and BP vary throughout the day in a circadian pattern and one hypothesis suggests the daily increases in HR and BP correspond to a recurrent rise in norepinephrine. This theory is supported by the fact that the incidence of ischemic events such as myocardial infarctions and stroke is highest in the morning. Numerous studies have evaluated the correlation between the time of day extended-release nifedipine is administered with NE levels and subsequent cardiovascular events (White et al 1998; Hermida et al 2007; Hermida et al 2008).

One double-blind, randomized, parallel-group trial of 557 patients compared controlled-onset extended-release (COER) verapamil $180 \mathrm{mg}$ taken at bedtime with nifedipine GITS $30 \mathrm{mg}$ taken in the morning for a maximum of 10 weeks (White et al 1998). Doses were increased in a step-wise fashion to a goal SBP $<140 \mathrm{mmHg}$ and DBP $<90 \mathrm{mmHg}$ (White et al 1998). The mean doses of nifedipine GITS and COER-verapamil at the end of the trial were $64 \mathrm{mg}$ and $314 \mathrm{mg}$, respectively (White et al 1998). The primary efficacy endpoint was the change in BP from baseline following four weeks of a consistent treatment dose (White et al 1998). Therapies were determined to be equal if the mean change from baseline was $\leq 5 \mathrm{mmHg}$ for SBP and $\leq 3 \mathrm{mmHg}$ for DBP (White et al 1998). After 10 weeks of treatment there was no significant difference in the mean change of early morning BP between treatment arms. There was a statistically significant difference in early morning HR between the two therapies ( $p<0.001$ ), however the clinical significance of the 4 beats/minute decrease with COER-verapamil as compared to the 2 beats per minute increase with nifedipine
GITS is minimal. The rate of rise in BP and HR in the early morning may be one of the more predictive factors in relation to cardiovascular events. This trial demonstrated that both therapies decreased the rate of increase in BP compared to baseline; however nifedipine GITS increased the rate of rise in HR while COER-verapamil significantly decreased the rate of rise in HR ( $p<0.001)$ (White et al 1998). The early morning HR-SBP product, an index of myocardial oxygen demand, was calculated after 4 and 10 weeks of treatment and showed that COER-verapamil significantly decreased the HR-SBP product compared with nifedipine GITS at 4 and 10 weeks ( $p<0.0001$ and $p=0.0003$, respectively). The greatest extent of lowering by COER-verapamil was between 4:00 AM and 8:00 PM. The largest difference occurred between 6:00 AM and 6:00 PM; the smallest difference was found between 10:00 PM and 4:00 AM. Twenty-four hour ambulatory BP values were also monitored after 4 and 10 weeks of therapy. Recordings demonstrated that 24 hour mean awake and sleep DBP were similar between groups (White et al 1998). This trial attempted to dose nifedipine GITS and COER-verapamil in a manner so that peak drug concentrations would be achieved at the time when BP and HR peak the most rapidly, in the early morning; however there was no significant difference in mean change in morning BP between groups (White et al 1998).

When $30 \mathrm{mg}$ of nifedipine GITS was administered to a small patient population there was no difference in the antihypertensive effect of the medication during the day or at night (Hermida et al 2007). A larger randomized, prospective, open-label trial of 80 patients was then conducted to determine if nifedipine GITS $30 \mathrm{mg}$ or $60 \mathrm{mg}$ given first thing in the morning or at bedtime would have varying effects on BP's circadian pattern over the course of 16 weeks (Hermida et al 2007). BP was measured over 48 hours via ABPM (Hermida et al 2007). The results showed that when nifedipine GITS $30 \mathrm{mg}$ was administered in the morning or at bedtime there was a significant decrease in 24-hour BP compared with baseline ( $\mathrm{p}<0.001$ for both regimens) (Hermida et al 2007). There was no effect on HR or the circadian pattern of $\mathrm{BP}$, including maximum $\mathrm{BP}$ readings throughout the 24-hour interval with either dosing strategy (Hermida et al 2007). In comparison, when $60 \mathrm{mg}$ doses of nifedipine GITS were administered, there was a significant benefit to bedtime dosing in relation to mean 24-hour BP control ( $p<0.001$ ) (Hermida et al 2007). Additionally, patients who received nifedipine GITS at bedtime reported significantly less edema than those who received the drug first thing in the morning $(p=0.026)$ (Hermida et al 2007). This trial demonstrated 
that the efficacy of nifedipine GITS is independent of the time of day at which it is administered; however there is a dose-dependent relationship relative to the drug's efficacy and safety with bedtime dosing as compared to early morning administration (Hermida et al 2007).

Another study by some of the same authors as the previously mentioned trial studied patients with hypertension who received nifedipine GITS $30 \mathrm{mg}$ daily either first thing in the morning or at bedtime for eight weeks (Hermida et al 2008). Because the earlier study showed a slight benefit in bedtime dosing, this theory was investigated further using ABPM (Hermida et al 2008). Both SBP and DBP were significantly decreased with bedtime dosing as compared to morning dosing (SBP $p=0.01$, DBP $p<0.001)$. Morning administration still decreased SBP and DBP significantly compared to baseline values (Hermida et al 2008). Asleep mean SBP, morning surge of SBP, and asleep mean DBP were significantly decreased with bedtime dosing as compared to morning dosing (Hermida et al 2008). The authors of this study concluded that bedtime administration of nifedipine GITS significantly improves ambulatory BP and the prevalence of edema compared to morning dosing (Hermida et al 2008).

\section{Tolerability}

Extended-release nifedipine appears to be relatively well tolerated, particularly compared with other antihypertensives, because it does not cause depression of the central nervous system or orthostasis (Gavras et al 1987). Common adverse events mentioned in the literature are summarized in Table 1. The most significant adverse effect, edema, is dose related and occurs in $10 \%$ to $30 \%$ of patients who are receiving $180 \mathrm{mg}$ (Pfizer 2003). When compared with placebo, headache and edema were more common in the nifedipine extended-release group (Pfizer 2003).

When nifedipine GITS was compared with COERverapamil the overall incidence of adverse effects was similar between groups ( $74 \%$ and $68 \%$, respectively) (White et al 1998). Peripheral edema (22\% vs $4 \%, \mathrm{p}<0.001)$ and arthralgias ( $6 \%$ vs $2 \%, \mathrm{p}=0.048)$ were reported significantly more often in the nifedipine GITS group compared to the COER-verapamil group (White et al 1998). When the GITS formulation was compared to prolonged action and capsule formulations nifedipine GITS was better tolerated with respect to overall adverse events, particularly headache and dizziness (Toal et al 2004). Only vomiting was more common in the nifedipine GITS arm compared to the other formulations (Toal et al 2004).

Nifedipine GITS was not as well tolerated as losartan/HCTZ or telmisartan/HCTZ in 12 week studies (Weir et al 1996; Fogari et al 2005). More patients in the nifedipine group withdrew from the study as compared to the losartan/HCTZ group (19\% vs 7\%, p = 0.012) (Weir et al 1996). The most common

Table I Incidence of adverse events reported in clinical trials

\begin{tabular}{|c|c|c|c|c|c|c|}
\hline Adverse event & $\begin{array}{l}\text { White et al } \\
1998 \\
\text { (mean } \\
64 \mathrm{mg} / \text { day) }\end{array}$ & $\begin{array}{l}\text { Toal et al } \\
2004 \\
\text { ( } 60 \mathrm{mg} / \text { day) }\end{array}$ & $\begin{array}{l}\text { Pfizer } 2003 \\
180 \text { mg/day }\end{array}$ & $\begin{array}{l}\text { Fogari et al } \\
2005 \\
60 \mathrm{mg} / \text { day }\end{array}$ & $\begin{array}{l}\text { Weir et al } \\
1996 \\
30,60 \text {, or } \\
90 \mathrm{mg} / \text { day }\end{array}$ & $\begin{array}{l}\text { Testa et al } \\
1998 \\
30 \mathrm{mg} / \text { day }\end{array}$ \\
\hline Overall incidence & $74 \%$ & $24 \%$ & & & $61 \%$ & \\
\hline Peripheral edema & $22 \%$ & & $10 \%-30 \%$ & $13.8 \%$ & $12 \%$ & $24.2 \%$ \\
\hline Constipation & $8 \%$ & & $3.3 \%$ & & & \\
\hline Arthralgia & $6 \%$ & & $<3 \%$ & & & \\
\hline Back pain & $1 \%$ & & $<1 \%$ & & & \\
\hline Headache & & $21 \%$ & $15.8 \%$ & $5.2 \%$ & $12 \%$ & $12.4 \%$ \\
\hline Dizziness & & $3 \%$ & $4.1 \%$ & $1.7 \%$ & $1 \%$ & \\
\hline Nausea & & $3 \%$ & $3.3 \%$ & $1.7 \%$ & & \\
\hline Vomiting & & $7 \%$ & $<1 \%$ & & & \\
\hline Increased alk phos & & & $5.4 \%$ & & & \\
\hline Fatigue & & & $5.9 \%$ & - & $2 \%$ & \\
\hline Polyuria & & & $<3 \%$ & $1.7 \%$ & & \\
\hline Rash erythematosus & & & $<3 \%$ & $1.7 \%$ & & \\
\hline Flushing & & & $<3 \%$ & $5.2 \%$ & & $8.4 \%$ \\
\hline Palpitation & & & $<3 \%$ & $3.4 \%$ & & \\
\hline Pruritus & & & $<3 \%$ & $1.7 \%$ & & \\
\hline
\end{tabular}


cause of withdrawal from the study was edema which was significantly more common in the nifedipine group than in those receiving losartan $(\mathrm{p}=0.005)$ (Weir et al 1996). Ankle edema (13.8\% vs $1.6 \%)$, headache $(5.2 \%$ vs $0 \%)$, dizziness $(1.7 \%$ vs $0 \%$ ), polyuria ( $1.7 \%$ vs $0 \%)$, rash erythematosus $(1.7 \%$ vs $0 \%$ ), flushing $(5.2 \%$ vs $0 \%)$, palpitation $(3.4 \%$ vs $0 \%)$, and pruritus $(1.7 \%$ vs $0 \%)$ were all more common in the nifedipine group compared to telmisartan-HCTZ (Fogari et al 2005).

Quality of life has been assessed in patients on different dihydropyridine CCBs. One such study investigated the change in quality of life from baseline assessed by the distress of side effects and symptoms of patients receiving either nifedipine GITS $30 \mathrm{mg}$ or amlodipine $5 \mathrm{mg}$ daily (Testa et al 1998). Patients were assessed using the Higher Symptom Distress Index score where higher scores were indicative of more distress (Testa et al 1998). Mean quality of life scores were comparable between groups at baseline (Testa et al 1998). Lower extremity edema $(24.2 \%$ vs $17.4 \%)$, flushing $(8.4 \%$ vs $10.7 \%)$, and headache $(12.4 \%$ vs $11.2 \%)$ were reported in $>5 \%$ of patients receiving either nifedipine or amlodipine with no difference between groups (Testa et al 1998). Fifteen percent of patients receiving nifedipine and $14 \%$ of patients receiving amlodipine withdrew before the study's completion due to adverse events (Testa et al 1998). Patients treated with nifedipine had more distress secondary to shortness of breath, constipation, and tachycardia as opposed to the amlodipine arm which had more distress as a result of thirst and loss of taste (Testa et al 1998). The Mental/Emotional Health scale improved significantly $(\mathrm{p}=0.012)$ from baseline in patients receiving nifedipine (Testa et al 1998). The Psychological Distress scale $(p=0.021)$, Anxiety subscale $(p=0.012)$, and Depression subscale $(\mathrm{p}=0.071)$ also improved from baseline; however the Depression subscale did not improve significantly (Testa et al 1998). In the amlodipine group, significant improvements were seen in the Mental/Emotional Health scale $(\mathrm{p}=0.038)$, Psychological Well-Being $(\mathrm{p}=0.042)$, and General Positive Affect subscale ( $p=0.037$ ); however there was a significant decrease in the Sexual Symptom Distress score $(p=0.045)$ (Testa et al 1998). In terms of the Quality of Life Summary scale, patients receiving nifedipine GITS showed a significant improvement in score $(p<0.05)$ while those receiving amlodipine did not change from baseline (Testa et al 1998). This trial showed that differences in quality of life scores may be attributable to the delivery system.

\section{Conclusion}

Clinicians have known for decades that nifedipine is effective at lowering BP. However, its use was curtailed when newer and seemingly safer options were introduced in the form of new drug classes and additional agents within the class of dihydropyridine CCBs. In the years that followed, several classes of antihypertensives were shown to provide a significant reduction in morbidity and mortality in high risk patients; this propelled these classes of drugs to the forefront of hypertension management, specifically ACEIs (Yusuf et al 2000), ARBs (Dahlof et al 2002), and $\beta$-blockers (The Capricorn investigators 2001). Thiazide diuretics have also proven their utility in managing hypertension patients and are widely accepted as a first-line option in patients devoid of a compelling reason to be prescribed another class of antihypertensive (The ALLHAT Officers and Coordinators for the ALLHAT Collaborative Research Group 2002; Chobanian et al 2003). Based on the clinical trial data described above, it appears that many of the concerns surrounding the older formulations of nifedipine have been addressed with the GITS formulation. Edema still appears to be relatively common, although timing of dosing may ameliorate this effect. While data are not yet available for long-term mortality benefit of long-acting nifedipine, it is reasonable to consider this medication in situations where other dihydropyridine CCBs would commonly be used (eg, as add-on therapy to improve the patient's likelihood of achieving their BP goal or as initial therapy in patients that need general coronary artery disease prevention and do not have a compelling reason for prescription of another class of antihypertensive). Aggressive use of long-acting nifedipine as a first-line antihypertensive is not supported by clinical data or current practice guidelines. A number of clinical trials evaluating long-acting nifedipine have recently been completed or are ongoing. These include trials evaluating the effects of nifedipine on NE, delivery of nifedipine via osmotically controlled-release oral delivery system (OROS), combination therapy with telmisartan, the effects of nifedipine on proteinuria and BP in patients with diabetes, the effect on renal function decline and efficacy compared with lisinopril. The results of these may provide additional insight into the most appropriate use of long-acting nifedipine.

\section{Disclosures}

The authors do not have any conflicts of interest related to the content of this publication.

\section{References}

Abernathy DR, Schwartz JB. 1999. Calcium-antagonist drugs. N Engl J Med, 341:1447-57.

Abrams J, Frishman WH, Bates SM, et al. 2001. Pharmacologic options for treatment of ischemic disease. In: Antman EM, (ed.) Cardiovascular Therapeutics: A Companion to Braunwald's Heart Disease. 2nd ed. Philadelphia, PA: WB Saunders: 768-95. 
Blood Pressure Lowering Treatment Trialists' Collaboration. 2003. Effects of different blood-pressure-lowering regimens on major cardiovascular events: results of prospectively-designed overviews of randomized trials. Lancet, 362:1527-35.

Brown MJ, Palmer CR, Castaigne A, et al. 2000. Morbidity and mortality in patients randomized to double-blind treatment with a long-acting calcium-channel blocker or diuretic in the international nifedipine GITS study: intervention as a goal in hypertension treatment (INSIGHT). Lancet, 356:366-72.

Champlain J, Karas M, Nguyen P, et al. 1998. Different effects of nifedipine and amlodipine on circulating catecholamine levels in essential hypertensive patients. J Hypertens, 16:1357-69.

Chung M, Reitberg DP, Gaffney M, Singleton W. 1987. Clinical pharmacokinetics of nifedipine gastrointestinal therapeutic system: a controlledrelease formulation of nifedipine. Am J Med, 83(suppl 6B):10-4.

Chobanian AV, Bakris GL, Black HR, et al. 2003. The seventh report of the Joint National Committee on Prevention, Detection, Evaluation, and Treatment of High Blood Pressure: The JNC 7 Report. JAMA, 289:2560-72.

Dahlof B, Devereux RB, Kjeldsen SE, et al. 2002. Cardiovascular morbidity and mortality in the Losartan Intervention For Endpoint reduction in hypertension study (LIFE): a randomized trial against atenolol. Lancet, 359:995-1003.

Fogari R, Mugellini A, Zoppi A, et al. 2003. Differential effects of lercanidipine and nifedipine GITS on plasma norepinephrine in chronic treatment of hypertension. Am J Hypertens, 16:596-9.

Fogari R, Preti P, Zoppi A, et al. 2005. Effect of telmisartan/hydrochlorothiazide combination versus nifedipine GITS on ambulatory blood pressure and sympathetic activation. Am J Hypertens, 18:577-83.

Frishman WH, Garofalo JL, Rothschild A, et al. 1987. Multicenter comparison of the nifedipine gastrointestinal therapeutic system and long-acting propranolol in patients with mild to moderate systemic hypertension receiving diuretics. Am J Med, 83(suppl 6B):15-9.

Frishman WH, Garofalo JL, Rothschild A, et al. 1989. The nifedipine gastrointestinal therapeutic system in the treatment of hypertension. Am J Cardiol, 64:65F-9F.

Gavras I, Mulinari R, Gavras H, et al. 1987. Antihypertensive effectiveness of the nifedipine gastrointestinal therapeutic system. Am J Med, 83(suppl 6B):20-3.

Gibbons RJ, Abrams J, Chatterjee K, et al. 2003. ACC/AHA 2002 guideline update for the management of patients with chronic stable anginasummary article: a report of the American College of Cardiology/ American Heart Association Task Force on practice guidelines (Committee on the Management of Patients With Chronic Stable Angina). J Am Coll Cardiol, 41:159-68.

Hermida RC, Ayala DE, Mojón A, et al. 2008. Chronotherapy with nifedipine GITS in hypertensive patients: improved efficacy and safety with bedtime dosing. Am J Hypertens, 21:948-54.

Hermida RC, Calvo C, Ayala DE, et al. 2007. Dose- and administration timedependent effects of nifedipine GITS on ambulatory blood pressure in hypertensive subjects. Chronobiol Int, 24(3):471-93.

Izzo JL, Jr, Levy D, Black HR. 2000. Clinical Advisory Statement: importance of systolic blood pressure in older Americans. Hypertension, 35:1021-4.

Lewington S, Clarke R, Qizilbash N, et al. 2002. Age-specific relevance of usual blood pressure to vascular mortality: a meta-analysis of individual data for one million adults in 61 prospective studies. Lancet, 360:1903-13.

MacMahon S, Peto R, Cutler J, et al. 1990. Blood pressure, stroke, and coronary heart disease. Part 1, prolonged differences in blood pressure: prospective observational studies corrected for the regression dilution bias. Lancet, 335:765-74.

Mancia G, De Backer G, Dominiczak A, et al. 2007. Guidelines for the management of arterial hypertension: The Task Force for the Management of Arterial Hypertension of the European Society of Hypertension (ESH) and of the European Society of Cardiology (ESC). J Hypertens, 25:1105-87.
Mancia G, Omboni S, Parati G. 2002. Twenty-four hour ambulatory blood pressure in the International Nifedipine GITS Study Intervention as a Goal in Hypertension Treatment (INSIGHT). J Hypertens, 20:545-53.

Mancia G, Ruilope LM, Palmer CR. 2004. Effects of nifedipine GITS and diuretics in isolated systolic hypertension - a subanalysis of the INSIGHT study. Blood Press, 13:310-5.

Neal B, MacMahon S, Chapman N. 1964. Effects of ACE inhibitors, calcium antagonists, and other blood-pressure-lowering drugs. Lancet, 356:1955-64.

Pfizer. Procardia XL (nifedipine extended release tablets): prescribing information (USA) [online]. 2003. Accessed 3 August 2008. URL: http://www.pfizer.com/files /products/uspi_procardia_xl.pdf.

Rosendorff C, Black HR, Cannon CP, et al. 2007. Treatment of hypertension in the prevention and management of ischemic heart disease: a scientific statement from the American Heart Association Council for High Blood Pressure Research and the Councils on Clinical Cardiology and Epidemiology and Prevention. Circulation, $115: 2761-88$.

Schulte K-L, Lenz T, Fischer M, et al. 2000. Underestimation of 24-hour hypotensive efficacy of nifedipine GITS versus enalapril: ambulatory recording as an adjunct to clinical blood pressure measurement. Blood Pressure, 9:221-6.

Shima E, Katsube M, Kato T, et al. 2008. Calcium channel blockers suppress cytokine-induced activation of human neutrophils. Am J Hypertens, 21:78-84.

Staessen JA, Wang JG, Thijs L. 2003. Cardiovascular prevention and blood pressure reduction: a quantitative overview updated until 1 March 2003. J Hypertens, 21:1055-76.

Swanson DR, Barclay BL, Wong PSL, Theeuwes F. 1987. Nifedipine gastrointestinal therapeutic system. Am J Med, 83(suppl 6B):3-9.

Testa M, Turner RR, Simonson DC, et al. 1998. Quality of life and calcium channel blockade with nifedipine GITS versus amlodipine in hypertensive patients in Spain. J Hypertens, 16:1839-47.

The ALLHAT Officers and Coordinators for the ALLHAT Collaborative Research Group. 2002. Major outcomes in high-risk hypertensive patients randomized to angiotensin-converting enzyme inhibitor or calcium channel blocker vs diuretic: The Antihypertensive and LipidLowering Treatment to Prevent Heart Attack Trial (ALLHAT). JAMA, 288:2981-97.

The Capricorn Investigators. 2001. Effect of carvedilol on outcome after myocardial infarction in patients with left-ventricular dysfunction: the CAPRICORN randomized trial. Lancet, 357:1385-90.

Toal CB. 2001. Blood pressure control in patients with mild to moderate essential hypertension switched from nifedipine gastrointestinal therapeutic system (GITS) $30 \mathrm{mg}$ to nifedipine GITS $20 \mathrm{mg}$. Clin Ther, 23:87-96.

Toal CB. 2004. Formulation dependent pharmacokinetics - does the dosage form matter for nifedipine? J Cardiovasc Pharmacol, 44:82-6.

Vasan RS, Beiser A, Seshadri S, et al. 2002. Residual life-time risk for developing hypertension in middle-aged women and men: The Framingham Heart Study. JAMA, 287:1003-10.

Weir MR, Elkins M, Liss C, et al. 1996. Efficacy, tolerability, and quality of life of losartan, alone or with hydrochlorothiazide, versus nifedipine GITS in patients with essential hypertension. Clin Ther, 18:411-27.

Wenzel RR, Allegranza G, Binggeli C, et al. 1997. Differential activation of cardiac and peripheral sympathetic nervous system by nifedipine: role of pharmacokinetics. J Am Coll Cardiol, 29:1607-14.

White WB, Black HR, Weber MA, et al. 1998. Comparison of effects of controlled onset extended release verapamil at bedtime and nifedipine gastrointestinal therapeutic system on arising on early morning blood pressure, heart rate, and the heart rate - blood pressure product. Am J Cardiol, 81:424-31.

Yusuf S, Sleight P, Pogue J, et al. 2000. Effects of an angiotensin-convertingenzyme inhibitor, ramipril, on cardiovascular events in high-risk patients. The Heart Outcomes Prevention Evaluation Study Investigators. NEngl $J$ Med, 342(3):145-53. 
